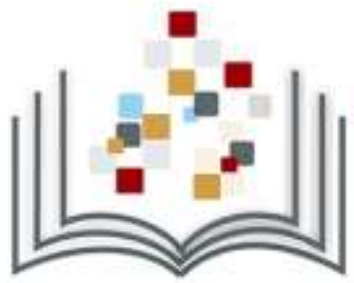

\title{
PROBLEMAS NA UTILIZAÇÃO DO OPEN JOURNAL SYSTEM (OJS/SEER)
}

Ronnie Fagundes de Brito ${ }^{1}$, Milton Shintaku ${ }^{1}$, Jaqueline Jesus ${ }^{1}$, Diego José Macedo ${ }^{1}$, Ingrid Torres Schiess ${ }^{1 *}$, Priscila Rodrigues dos Santos ${ }^{1}$, Priscila Paiva de Castro ${ }^{1}$, Andrea Maria C. S. F. Curado ${ }^{1}$, Lucas Angelo ${ }^{1}$, Lucas Rodrigues ${ }^{1}$

${ }^{1}$ Coordenação de Articulação, Geração e Aplicação de Tecnologia - Instituto Brasileiro de Informação em Ciência e Tecnologia - Brasília (DF) - Brasil

*Autor correspondente: ingridschiessl@ibict.br

\section{Como citar:}

BRITO, Ronnie F. et al.. Problemas na utilização do open journal system (ojs/seer). In: WORKSHOP DE EDITORAÇÃO CIENTÍFICA, 9., 2016, São Paulo. Anais... São Paulo:

Associação Brasileira de Editores Científicos, 2016. p. 7-12 DOI: http://dx.doi.org/10.21452/wec.IXwec.2016.0002

\section{RESUMO}

O Brasil é um grande usuário da ferramenta livre Open Journal System (OJS) para criação de periódicos científicos. Entretanto, essa tecnologia não está totalmente absorvida por parte dos usuários, muito provavelmente, pela dinamicidade da informática. Assim, levanta-se os problemas enfrentados pelos usuários do OJS no Brasil, verificando quais as dificuldades enfrentadas pelos profissionais que utilizam a ferramenta nos processos editoriais. Adotou-se na coleta de documentos as bases de dados das postagens do fórum, relacionadas às consultas sobre o OJS, obtendo variáveis quantitativas e qualitativas, baseando-se em estudos informétricos. Nos resultados, revelam-se o perfil dos usuários e apresentam quais os problemas mais comuns, do Fórum. Conclui-se que apesar das limitações, a opção do Fórum se apresenta como uma boa forma de interação e armazenamento de soluções, possuindo informações que apoiam a transferência de conhecimento. A principal contribuição desta pesquisa será subsidiar as atualizações de documentações técnicas.

\section{INTRODUÇÃO}

O Open Journal System (OJS) é uma ferramenta livre, de código aberto, muito utilizado no Brasil, em parte pelo apoio do Instituto Brasileiro de Informação em Ciência e Tecnologia (lbict). Esse software é mantido pelo Public Knowledge Project (PKP), recebendo contribuições provenientes de todo o mundo, principalmente nas traduções, visto que a ferramenta está traduzida para línguas minoritárias como galês, vietnamita, malaio, entre outras. No Brasil, foi traduzido pelo lbict e nomeado Sistema Eletrônico de Editoração de Revistas (SEER), sendo amplamente utilizado em diferentes revistas científicas nacionais, conforme Shintaku, Brito, e Carvalho Neto (2014).

Williams e Dew (2012) atribuem o grande uso do OJS por ser gratuito, dado os problemas financeiros que as revistas enfrentam, principalmente as de acesso aberto. Esse ponto concorda com Freire e Souza (2010), afirmando que o OJS é uma solução prática e econômica, na medida em que atende a necessidade de redução de custos, com eficiência nos processos editoriais. Assim, como defendem Bergamaschi e Duarte (2012), o OJS se apresenta como um modelo alternativo para ampliar o acesso, visibilidade, impacto e preservação dos artigos publicados. 
O OJS possibilita, além de criar uma revista, estabelecer um portal com todas as revistas que sejam de interesse da instituição. Ao hospedar várias revistas em uma única instalação, oferece maior economia na manutenção das revistas. Nesse sentido, Moraes, Miranda e Lima (2010) destacam que o OJS fomenta a qualidade das revistas, pois ajuda a padronizar as revistas hospedadas no portal.

Entretanto, essa ferramenta ainda apresenta alguns problemas de usabilidade relacionados à etapa de submissão, expostos por Brito e Shintaku (2015). Revela-se, assim, a necessidade de estudos mais amplos, destinados a análise de todas as facetas que envolvem esse software, na medida em que foi desenvolvido para o cenário de publicações de acesso aberto norte-americano.

No Brasil, o lbict assiste ao uso do OJS desde 2005, oferecendo apoio às equipes editorial e de informática de universidades, associações e outras entidades que disponibilizam publicações científicas, tecnológicas e outras intensivas em conhecimento. Essa assistência dá-se, interativamente, por meio de consultas via $e$ mails e uso do Fórum on-line ${ }^{1}$. E de forma não interativa, o Instituto produz e dissemina informação técnica sobre o OJS por meio de publicação e páginas no formato de Wikipédia.

Nesse contexto, o presente estudo tem por objetivo levantar e verificar as dificuldades manifestadas pelos os usuários do OJS, no Brasil, expostas no Fórum. Bem como, apresentar insumos para a tomada de decisão de quais temas serão tratados nas documentações técnicas desenvolvidas pelo lbict e qual a forma de tratamento das informações.

\section{METODOLOGIA}

Utiliza-se das técnicas de pesquisa documental nas bases de dados das postagens do fórum, relacionadas às consultas sobre o OJS. Considera-se assim cada postagem como um documento. Dessa forma, demonstrando ser um estudo de caráter humano e social, como afirmado por Sá-Silva, Almeida e Guindani (2009) mais comuns nas pesquisas documentais.

Possui alinhamentos com os estudos informétricos, dado que, no levantamento de variáveis quantitativas - a quantidade de postagens, além das qualitativas - o conteúdo das postagens, pois como afirma Macias-Chapula (1998) a informetria é o estudo de aspectos quantitativos em documentos em qualquer formato, para avaliar informação.

\section{RESULTADOS E DISCUSSÕES}

O estudo foi realizado com as 132 postagens do Fórum, no período de maio de 2015 a junho de 2016, ou seja, abrangendo um ano de postagens, sendo considerado um espaço temporal significativo para o estudo. Com isso, o estudo alinha ao que De Oliveira (2005) afirma, defendendo que fóruns são bons instrumentos para transferência de conhecimento de forma colaborativa.

Esse ponto pode ser confirmado pelas interações e visualizações das postagens. As 132 postagens tiveram 337 interações, ou seja, estas se dão por meio dos comentários às postagens, tais como réplicas e tréplicas. As visualizações foram ainda mais significativas, foram 35.347 acessos, revelando o interesse nas discussões sobre problemas, podendo ter solucionado questões semelhantes.

\footnotetext{
${ }^{1}$ http://forum.ibict.br/
} 


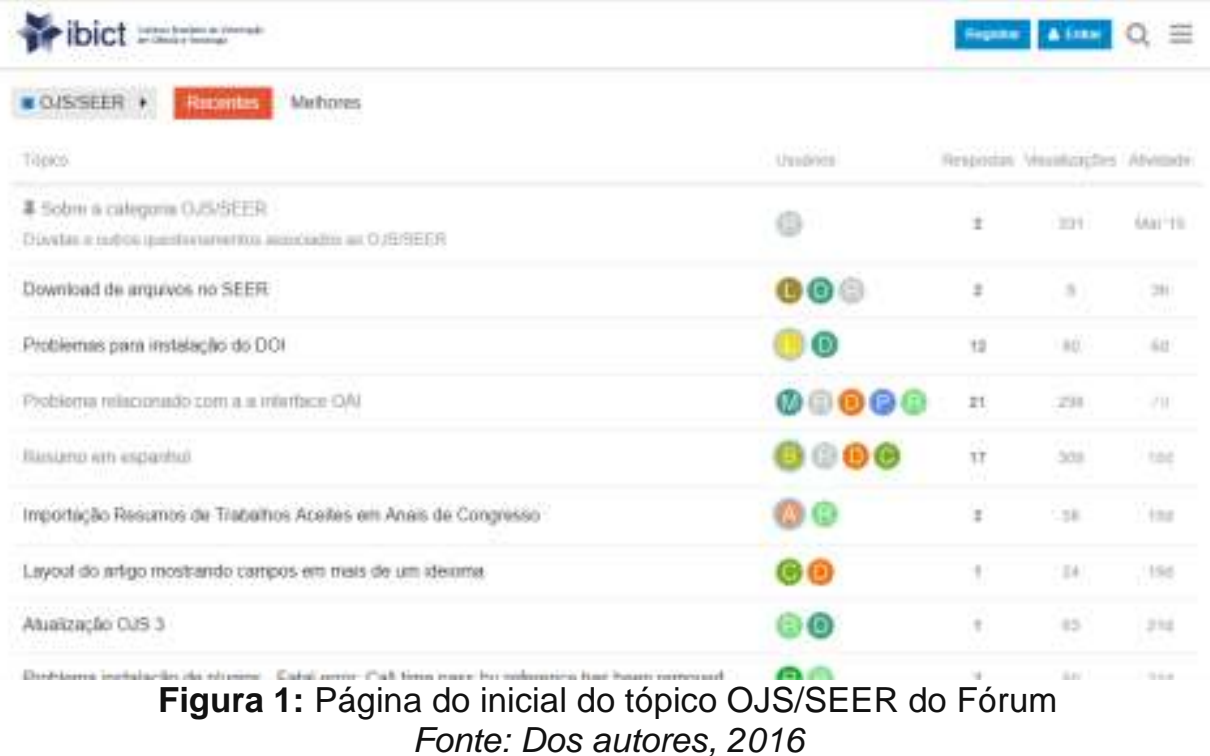

Numa primeira classificação, verificou-se distintamente duas categorias de postagens: técnica e tecnológica. A primeira, apresenta como assunto principal as dúvidas em relação ao uso. E a segunda, referem-se as questões sobre instalação, configurações iniciais elou atualizações, como apresentado no gráfico 1. É possível verificar uma maior quantidade de atividade relacionada aos problemas técnicos em todos os quesitos. Isto demonstra evidências de uso maior do fórum por parte dos editores ou auxiliares de editores, que dos profissionais de informática.

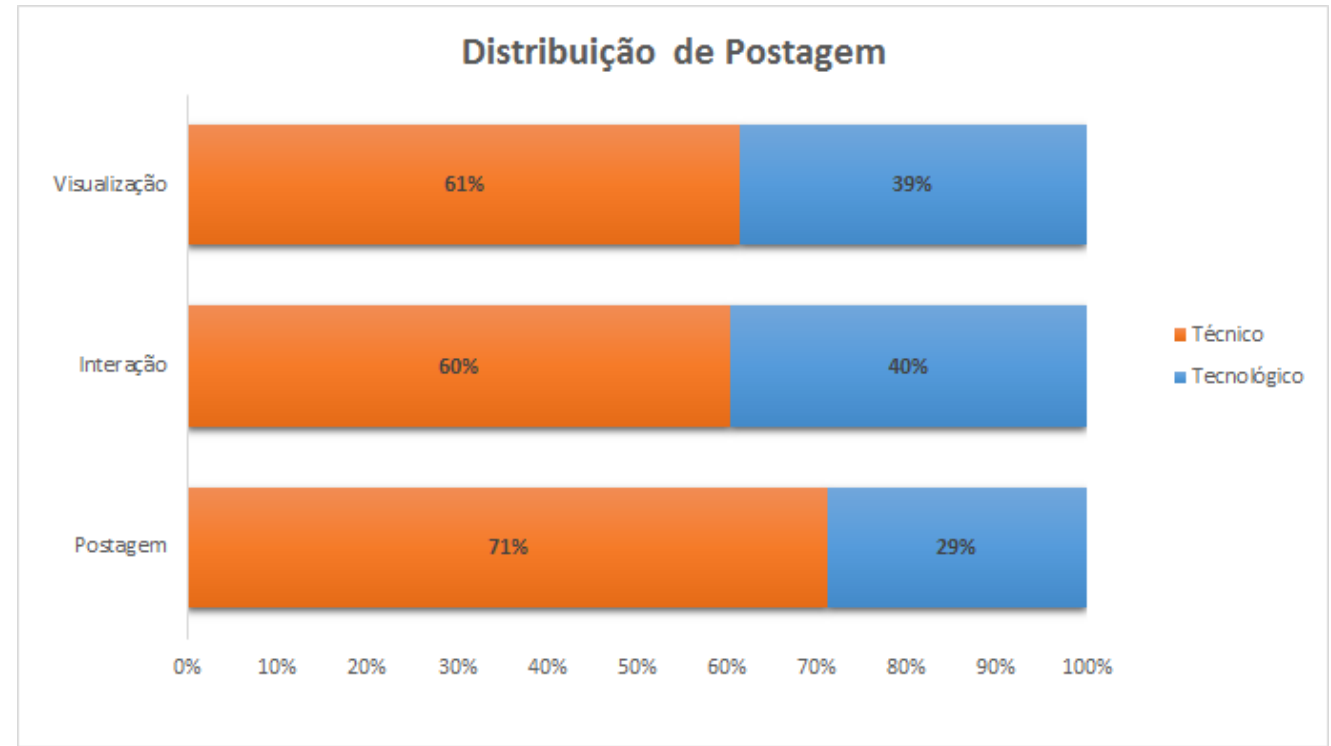

Gráfico 1: Distribuição de postagens

Fonte: Dos autores, 2016.

Nas postagens relacionadas aos problemas tecnológicos, a classe com o maior número de postagens e interações é a denominada Configurações, com 16 postagens, 77 interações e 7.127 visualizações. Esses pontos apresentam indícios que os usuários ainda não possuem grande familiaridade com o OJS, com maior dúvidas na customização dessa ferramenta, em uma categoria que envolve, em alguns casos, os responsáveis técnicos da revista. No Quadro 1, alguns exemplos de postagens classificada como tecnológica: 
Usuário 1:"Estamos tendo problemas em inserir a revista no Dialnet, devido a um problema com o OAl. Parece que o OAl da revista não está respondendo adequadamente (um problema relacionado com a não apresentação de metadados). Qual o caminho para resolver o problema?"

Usuário 2: "posso criar um banco de dados com outro nome diferente do default esperado pelo sistema que é o ojs.

Vi que esse é o nome default que ele usa para criar o banco de dados e o usuário também. Posso criar por exemplo, ojs_outronome?",

Usuário 3: "Precisamos de ajuda para tornar o campo afiliação obrigatório no momento de uma nova submissão. Precisamos de uma linha de código que resolva o problema. A parte visual, que apresenta 0 * no campo está OK, o que precisamos é do código que torne o campo realmente obrigatório."

Quadro 1: Postagens classificadas como tecnológica Fonte: Dos autores, 2016.

Nas questões técnicas, por sua vez, há uma grande variedade de assuntos tratados. Tanto que, grande parte das postagens não foram passíveis de classificação. Os problemas com e-mails e usuários se apresentaram como os mais frequentes. Destaque para as postagens relacionadas ao Digital Object Identifier (DOI), que foram as mais comentadas e visualizadas, revelando a necessidade de maiores esclarecimentos sobre o tema. Foram apenas quatro postagens sobre o DOI, que levaram a 19 interações e 1.077 visualizações. No Quadro 2, alguns exemplos de postagens classificada como técnicas:

Usuário 4: "Gostaria de saber se tem como incluir outros tipos de usuário, além dos editores, editor gerente, etc. Se sim, como incluímos?"

Usuário 5: "Uma dúvida, caso um artigo tenha sido exportado do OJS/SEER para inclusão de DOI e neste faltou o cadastro de um autor, e este após o DOI estar validado na CrossRef, e incluirmos ele nos metadados do artigo no SEER, ele não consegue incluir em seu Lattes o artigo pelo DOI?"

Usuário 6: "é possível personalizar o texto do e-mail de agradecimento para os novos usuários? E também, é possível alterar as características do texto (tamanho/fonte) das páginas da plataforma?"

\section{Quadro 2: Postagens classificadas como técnica}

Fonte: Dos autores, 2016.

\section{CONCLUSÃO}

O presente estudo teve por finalidade analisar as postagens expostas no Fórum na categoria OJS/Seer do Ibict a fim de verificar as dificuldades dos usuários do OJS no processo editorial. Entretanto, mesmo com as limitações do Fórum, Batista e Gobara (2007) defende que num processo de disseminação do conhecimento, os fóruns ainda se apresentam como uma boa ferramenta de interação e armazenamento de soluções.

A abrangência das postagens, verificadas pela quantidade de visualizações, revelam a capacidade do fórum em disponibilizar informações que apoiem a transferência de conhecimento. Da mesma forma, em que as interações permitem dialogar com outros usuários do fórum na busca por soluções dos problemas.

Por fim, revelou-se que o perfil dos usuários do fórum são mais técnicos do que tecnológicos, apresentando os problemas mais comuns. Assim, pode-se utilizar essa informação como apoio para definir quais os temas serão tratados nas documentações desenvolvidas pelo lbict, tais como, os guias e manuais, as cartilhas, as atualizações da Wiki, etc. Igualmente, orientar a forma de tratamento das informações disponibilizadas. 


\section{REFERÊNCIAS}

BATISTA, Erlinda M.; GOBARA, Shirley T. O fórum on-line e a interação em um curso a distância. Renote, v. 5, n. 1, 2007. Disponível em:

$<$ http://seer.ufrgs.br/index.php/renote/article/view/14248/8162>. Acesso em: 18 mar. 2015.

BERGAMASCHI, Rafael. J. P.; DUARTE, Raquel. B. P.. O Seer e o processo de editoração de revistas científicas brasileiras. In: ENCONTRO VIRTUAL DE DOCUMENTAÇÃO EM SOFTWARE LIVRE E CONGRESSO INTERNACIONAL DE LINGUAGEM E TECNOLOGIA ONLINE. 1., 2012, Belo Horizonte. Anais... Belo Horizonte: UFMG, 2012. Disponível em:<

http://www.periodicos.letras.ufmg.br/index.php/anais linguagem tecnologia/article/vie w/1943/3554 >. Acesso em: 15 jan. 2014.

BRITO, Ronnie F. de; SHINTAKU, Milton. Usability of the Submission Process in a Journal System. In: INTERNATIONAL CONFERENCE ON HUMAN-COMPUTER INTERACTION. 17., 2015, Los Angeles. Proceedings..., part II. Los Angeles: Springer International Publishing, 2015. Disponível em: <

http://link.springer.com/chapter/10.1007\%2F978-3-319-21383-5 109 >. Acesso em: 20 maio 2015.

DE OLIVEIRA, Gerson P.. O fórum em um ambiente virtual de aprendizado colaborativo. Revista de Tecnologia Digital e Educação a Distância, v. 2, n. 1, 2005. Disponível em: < http://www4.pucsp.br/tead/n2/pdf/artigo3.pdf >. Acesso em: 10 jun. 2016.

FREIRE, Isa M.; SOUZA, Alexandre P. Revista Pesquisa Brasileira em Ciência da Informação e Biblioteconomia - PBCIB: um mapeamento temático da produção científica à luz da análise de conteúdo. Informação \& Informação, [S.I.], v. 15, n. 2, p. 110-128, dez. 2010. Disponível em: <

http://www.uel.br/revistas/uel/index.php/informacao/article/view/5338 >. Acesso em: 2 Set. 2015.

MACIAS-CHAPULA, Cesar A. O papel da informetria e da cienciometria e sua perspectiva nacional e internacional. Ciência da informação, v. 27, n. 2, p. 134-140, 1998. Disponível em: < http://revista.ibict.br/ciinf/article/view/794/825 > . Acesso em: 10 set. 2015.

MORAES, Maria; MIRANDA, Angélica; LIMA, Jeane. Portal de Periódicos da FURG, acesso aberto e ampliação da produção científica institucional. In: ENCONTRO NACIONAL DE USUÁRIOS DO SEER, 1., 2010, Santa Catarina. Anais... Santa Catarina: Ibict, 2010. Disponível em: <

http://euseer.ibict.br/index.php/iieuseer/euseer1/paper/view/34 >. Acesso em: 5 Set. 2015.

SÁ-SILVA, Jackson R.; DE ALMEIDA, Cristóvão D.; GUINDANI, Joel F.. Pesquisa documental: pistas teóricas e metodológicas. Revista Brasileira de História \&

Ciências Sociais, [S.I.], v. 1, n. 1, 2009. Disponível em:< https://www.rbhcs.com/rbhcs/article/view/6/pdf >. Acesso em: 5 Set. 2015. 
WILLIAMS, Joe. M.; DEW, Stephen. H. Creating a Open-Access, Peer-Reviewed Journal: The Journal of Learning Spaces in: KRAUTTER, M. et al. In: The Entrepreneurial Librarian: Essays on the Infusion of Private-Business Dynamism into Professional Service. North Carolina: McFarland, 2012.

PALAVRAS-CHAVE: Comunicação científica. Open Journal System. Fórum. 